\title{
Lens thickness and insulin dependent diabetes mellitus: a population based twin study
}

\author{
N Logstrup, A K Sjølie, K O Kyvik, A Green
}

\begin{abstract}
Aim-To investigate the relation between lens thickness and duration of insulin dependent diabetes mellitus (IDDM).

Methods-From the new population based Danish twin register, containing 20888 twin pairs born between 1953 and 1982 (inclusive), all twin pairs having one or both partners affected with IDDM were searched. Among the $\mathbf{4 5}$ twin pairs available for clinical eye examination there were 15 monozygotic pairs, 14 dizygotic pairs of same sex, and 16 dizygotic pairs of opposite sex. Lens thickness was measured by ultrasonography. Using a twin control design, the relation between lens thickness and duration of IDDM was assessed by estimating the correlation between the intrapair difference in lens thickness and the intrapair difference in diabetes duration.
\end{abstract}

Results-In monozygotic twin pairs a statistically highly significant correlation between duration of diabetes and lens thickness was found (right eye: $r=0.88$, $\mathbf{p}<0.0001$; left eye: $r=0.90, p<0.0001)$. In dizygotic twin pairs of the same sex the correlations were $r=0.58(p=0.029)$ and $r=0.53(p=0.053)$ for right eye and left eye, respectively. For dizygotic twin pairs of opposite sex the correlations were $r=0.58$ $(p=0.018)$ and $r=0.69(p=0.005)$ for right eye and left eye, respectively. The slope in regression analysis were similar for monozygotic twin pairs $(0.025$, common for both eyes) and dizygotic twin pairs grouped (0.024, common for both eyes). Conclusions-There is a statistically significant positive correlation between duration of IDDM and lens thickness, as assessed by the twin control method. The higher correlation in monozygotic twins compared with dizygotic twins suggests that genetic factors play an additional role in the determination of lens thickness. The similar slopes in regression analysis indicate that the effect of diabetes duration on lens thickness is independent of zygosity.

(BrF Ophthalmol 1996; 80: 405-408)

The genetic influence on refraction and its components has been investigated previously in twin studies. ${ }^{1-5}$ Refraction is also influenced by environmental factors and disease conditions. ${ }^{6}$ Thus, diabetes mellitus may affect refraction with short term fluctuations and more permanent alterations. The generally accepted view is that short term fluctuations alter the refraction of the lens, primarily by alterations in osmotic pressure caused by changes in the blood glucose level. There is no general agreement regarding the direction of these refractive changes. ${ }^{8}$ It has been suggested that there is a higher degree of myopia, when there is a high blood glucose level, and a hyperopic shift when the blood glucose level normalises. ${ }^{910}$ Other studies, however, suggest alterations in a hyperopic direction at high blood glucose levels. ${ }^{11-13}$ Furthermore, some investigations have shown an increased prevalence of low degree myopia among diabetic patients. ${ }^{1415}$

The lens is a major modifiable determinant of refraction. Several reports have shown increased lens thickness in diabetic patients. ${ }^{16-18}$ Since lens thickness increases with age, it is difficult to separate the effect of diabetes and, particularly, diabetes duration from the effect of increasing age.

Investigations in twins offer unique opportunities for eliminating the effects of age and other confounding factors in studies of the association between diabetes duration and lens thickness. Partners of monozygotic (MZ) twin pairs are genetically identical and matched for age and most environmental influences that determine growth and development. Partners of dizygotic (DZ) twin pairs have the same features, except for sharing only $50 \%$ of their genes on average. The twin control method utilises intrapair differences in exposures (diabetes duration) and outcomes (lens thickness) to eliminate confounding factors in the assessment of a possible association between exposure and outcome.

We here report the results of a twin control study of the association between duration of IDDM and lens thickness. The twin pairs have been ascertained from the new part of the Danish twin register ${ }^{19}$ as part of a larger twin study on IDDM. ${ }^{20}$

\section{Material and methods}

TWIN SAMPLE

The new part of the Danish twin register includes 20888 twin pairs, born in Denmark 1953-82 (inclusive). It has been established on the basis of the Danish Civil Registration System and is considered a representative sample comprising $74.4 \%$ of all Danish twin pairs born 1953-67, and virtually complete (97.4\%) concerning twin pairs born 1968-82. ${ }^{19}$ Among 19180 twin pairs responding to a questionnaire survey on IDDM,, 20102 pairs had one or both partners affected with
IDDM. Of these, 54 pairs were not available
Correspondence to: Niels Logstrup, Department of Ophthalmology, Vejle Denmark.

Accepted for publication 7 February 1996 
Table 1 Summary of study material

Twin pairs born 1953-82 registered in the new Danish

twin register
Twin pairs with IDDM identified by questionnaire

One or both unwilling to participate

One or both unwilling to partner had died

One twin partner had emigrated

Non-response in one twin partne

Twin pairs investigated

Did not want pupils dilated

Did not cooperate to ultrasonic measurements

Twin pairs in analysis

*Among these, one left eye was excluded because of aphakia after trauma.

for further examination. Among the remaining 48 pairs, 45 were included for analysis in the present study (Table 1).

The diagnosis of IDDM has been based on internationally accepted clinical criteria combined with no or low fasting $\mathrm{C}$ peptide. ${ }^{20}$ Onset of diabetes was defined as the month and year when the first insulin injection was given. The zygosity diagnosis was established on 11 blood and enzyme type systems, providing a more than $99 \%$ reliable classification. ${ }^{20}$

Table 2 Age, sex, duration of diabetes, and intrapair difference in diabetic duration, as well as lens thickness and intrapair difference in lens thickness, for all the twins in analysis

\begin{tabular}{|c|c|c|c|c|c|c|c|c|c|c|c|}
\hline \multirow{2}{*}{$\begin{array}{l}\text { Twin } \\
\text { pair } \\
\text { No }\end{array}$} & \multirow{2}{*}{$\begin{array}{l}\text { Age } \\
\text { (years) }\end{array}$} & \multirow[b]{2}{*}{ Sex } & \multicolumn{2}{|c|}{$\begin{array}{l}\text { Duration of } \\
\text { IDDM (years) }\end{array}$} & \multirow{2}{*}{$\begin{array}{l}\text { Difference } \\
\text { in IDDM } \\
\text { duration } \\
(A-B)\end{array}$} & \multicolumn{4}{|c|}{$\begin{array}{l}\text { Lens thickness } \\
(\mathrm{mm})\end{array}$} & \multicolumn{2}{|c|}{$\begin{array}{l}\text { Difference in } \\
\text { lens thickness }\end{array}$} \\
\hline & & & $A$ & $B$ & & $R(A)$ & $L(A)$ & $R(B)$ & $L(B)$ & $R d i f$ & Ldif \\
\hline \multicolumn{12}{|l|}{ MZ: } \\
\hline 1 & $14 \cdot 8$ & $\mathbf{M}$ & 0.3 & - & 0.3 & 3.46 & 3.52 & 3.49 & 3.62 & -0.03 & -0.10 \\
\hline 2 & 39.7 & $\mathbf{M}$ & $17 \cdot 6$ & $16 \cdot 6$ & $1 \cdot 0$ & 3.72 & 3.72 & 3.84 & 3.79 & $-0 \cdot 12$ & -0.07 \\
\hline 3 & $25 \cdot 3$ & M & 3.0 & - & 3.0 & $4 \cdot 04$ & $4 \cdot 14$ & 4.02 & 4.04 & 0.02 & 0.10 \\
\hline 4 & 36.8 & $\mathbf{M}$ & $3 \cdot 4$ & 0.3 & $3 \cdot 1$ & $4 \cdot 16$ & $4 \cdot 27$ & 4.08 & 4.03 & 0.08 & $0 \cdot 24$ \\
\hline 5 & $24 \cdot 5$ & $\mathrm{~F}$ & $3 \cdot 4$ & - & 3.4 & $3 \cdot 85$ & 3.85 & 3.72 & 3.83 & 0.13 & 0.02 \\
\hline 6 & $31 \cdot 7$ & M & 18.9 & $15 \cdot 1$ & $3 \cdot 8$ & $4 \cdot 10$ & $4 \cdot 29$ & 4.03 & 4.09 & 0.07 & 0.20 \\
\hline 7 & 36.8 & $\mathbf{M}$ & 13.9 & $9 \cdot 1$ & $4 \cdot 8$ & 3.75 & 3.75 & 3.82 & $3 \cdot 74$ & -0.07 & 0.01 \\
\hline 8 & $25 \cdot 2$ & $\mathbf{M}$ & 5.7 & - & $5 \cdot 7$ & 3.73 & 3.66 & 3.63 & $3 \cdot 70$ & $0 \cdot 10$ & -0.04 \\
\hline 9 & $30 \cdot 1$ & $\mathbf{F}$ & 6.0 & - & $6 \cdot 0$ & 3.77 & 3.87 & 3.73 & 3.79 & 0.04 & 0.08 \\
\hline 10 & $31 \cdot 0$ & $\mathbf{M}$ & 13.8 & $5 \cdot 8$ & $8 \cdot 0$ & $3 \cdot 64$ & 3.65 & $3 \cdot 70$ & 3.61 & -0.06 & 0.04 \\
\hline 11 & $11 \cdot 7$ & $\mathbf{M}$ & 8.7 & - & $8 \cdot 7$ & 3.63 & 3.58 & 3.45 & 3.47 & $0 \cdot 18$ & 0.11 \\
\hline 12 & $32 \cdot 2$ & M & $17 \cdot 2$ & $7 \cdot 1$ & $10 \cdot 1$ & 3.92 & 3.93 & 3.71 & $3 \cdot 78$ & 0.21 & 0.15 \\
\hline 13 & 19.9 & $\mathbf{F}$ & $10 . \overline{7}$ & - & 10.7 & $3 \cdot 74$ & 3.77 & 3.48 & 3.55 & 0.26 & $0 \cdot 22$ \\
\hline 14 & $38 \cdot 5$ & $\mathbf{F}$ & $26 \cdot 7$ & - & $26 \cdot 7$ & $4 \cdot 19$ & $4 \cdot 19$ & 3.83 & $3 \cdot 71$ & 0.36 & 0.48 \\
\hline 15 & $35 \cdot 0$ & $\mathbf{M}$ & $30 \cdot 1$ & - & $30 \cdot 1$ & 4.41 & $4 \cdot 26$ & 3.46 & 3.46 & 0.95 & 0.80 \\
\hline \multicolumn{12}{|c|}{ DZss: } \\
\hline 1 & $25 \cdot 8$ & $\mathbf{M}$ & $2 \cdot 0$ & - & $2 \cdot 0$ & 3.41 & 3.20 & 3.48 & 3.39 & -0.07 & -0.19 \\
\hline 2 & $37 \cdot 1$ & $\mathbf{M}$ & $2 \cdot 6$ & - & $2 \cdot 6$ & $4 \cdot 12$ & $4 \cdot 14$ & $4 \cdot 21$ & $4 \cdot 23$ & -0.09 & -0.09 \\
\hline 3 & $29 \cdot 2$ & $\mathrm{~F}$ & $3 \cdot 2$ & - & $3 \cdot 2$ & 3.60 & 3.59 & 3.66 & 3.66 & -0.06 & -0.07 \\
\hline 4 & $37 \cdot 6$ & $\mathbf{M}$ & 3.6 & - & $3 \cdot 6$ & $3 \cdot 86$ & 3.93 & 3.66 & 3.73 & 0.20 & 0.20 \\
\hline 5 & $34 \cdot 4$ & $\mathrm{~F}$ & $3 \cdot 8$ & - & $3 \cdot 8$ & 3.90 & 3.95 & 3.86 & 3.94 & 0.04 & 0.01 \\
\hline 6 & $37 \cdot 3$ & M & $4 \cdot 0$ & - & $4 \cdot 0$ & 4.05 & 4.03 & $4 \cdot 16$ & $4 \cdot 13$ & $-0 \cdot 11$ & $-0 \cdot 10$ \\
\hline 7 & 11.6 & $\mathrm{~F}$ & $4 \cdot 6$ & - & $4 \cdot 6$ & $3 \cdot 42$ & 3.36 & $3 \cdot 32$ & $3 \cdot 31$ & $0 \cdot 10$ & 0.05 \\
\hline 8 & 35.6 & M & 6.8 & - & $6 \cdot 8$ & 3.75 & 3.7 & 3.97 & 3.91 & -0.22 & -0.20 \\
\hline 9 & $34 \cdot 8$ & $\mathbf{M}$ & $11 \cdot 2$ & - & $11 \cdot 2$ & $4 \cdot 11$ & $4 \cdot 21$ & 3.98 & 4.04 & 0.13 & $0 \cdot 17$ \\
\hline 10 & $16 \cdot 8$ & $\mathrm{~F}$ & 12.6 & - & $12 \cdot 6$ & $3 \cdot 80$ & 3.96 & $3 \cdot 46$ & 3.35 & 0.34 & 0.61 \\
\hline 11 & $24 \cdot 1$ & M & 13.8 & - & 13.8 & $4 \cdot 2$ & $4 \cdot 1$ & 3.92 & 3.95 & 0.31 & 0.20 \\
\hline 12 & $18 \cdot 3$ & $F$ & $14 \cdot 4$ & - & 14.4 & $3 \cdot 77$ & 3. & & 3. & 0.07 & 0.02 \\
\hline 13 & $20 \cdot 7$ & $\mathrm{~F}$ & $15 \cdot 7$ & - & $15 \cdot 7$ & 3.91 & $4 \cdot($ & 3. & $3 \cdot 78$ & 0.23 & 0.25 \\
\hline 14 & $24 \cdot 9$ & $\mathrm{~F}$ & 16.9 & - & 16.9 & 3.87 & $3 \cdot \varepsilon$ & & 3.81 & 0.07 & 0.02 \\
\hline \multicolumn{12}{|l|}{ DZos: } \\
\hline 1 & $22 \cdot 8$ & FM & $2 \cdot 3$ & - & $2 \cdot 3$ & 3.63 & 3.68 & 3.80 & $3 \cdot 81$ & $-0 \cdot 17$ & -0.13 \\
\hline 2 & $27 \cdot 6$ & FM & $2 \cdot 6$ & - & $2 \cdot 6$ & $3 \cdot 74$ & 3.66 & 3.86 & 3.87 & $-0 \cdot 12$ & -0.21 \\
\hline 3 & 32.9 & FM & $2 \cdot 8$ & - & $2 \cdot 8$ & 3.68 & $3 \cdot 60$ & 3.66 & 3.65 & 0.02 & -0.05 \\
\hline 4 & $36 \cdot 6$ & MF & $3 \cdot 2$ & - & $3 \cdot 2$ & $4 \cdot 01$ & 4.06 & 4.51 & & -0.50 & * \\
\hline 5 & $31 \cdot 4$ & FM & 4.9 & - & 4.9 & 3.67 & 3.69 & 3.76 & 3.97 & -0.09 & -0.28 \\
\hline 6 & 26 . & M & $5 \cdot 7$ & - & $5 \cdot 7$ & 3.48 & 3.52 & 3.69 & 3.69 & $-0 \cdot 21$ & -0.17 \\
\hline 7 & 22.4 & FN & $7 \cdot 3$ & - & $7 \cdot 3$ & 3.39 & & & & $-0 \cdot 14$ & -0.01 \\
\hline 8 & $15 \cdot 1$ & FM & $7 \cdot 7$ & - & $7 \cdot 7$ & $3 \cdot 72$ & 3.70 & 3.81 & 3.85 & -0.09 & -0.15 \\
\hline 9 & $14 . C$ & M & $7 \cdot 9$ & - & 7.9 & $3 \cdot 67$ & 3.56 & & & 0.29 & 0.11 \\
\hline 10 & 35.5 & FM & $29 \cdot 8$ & $19 \cdot 0$ & $10 \cdot 8$ & $4 \cdot 75$ & $4 \cdot 87$ & $4 \cdot 32$ & $4 \cdot 37$ & 0.43 & 0.50 \\
\hline 11 & $27 \cdot 9$ & FM & 11.9 & - & 11.9 & 3.80 & 3. & & 3.51 & 0.36 & 0.30 \\
\hline 12 & $26 \cdot 7$ & MF & 14.6 & - & $14 \cdot 6$ & $3 \cdot 86$ & 3.92 & 3.80 & 3.79 & 0.06 & 0.13 \\
\hline 13 & $28 \cdot 8$ & FM & $15 \cdot 2$ & - & $15 \cdot 2$ & $4 \cdot 40$ & $4 \cdot 40$ & & 3.97 & 0.48 & 0.43 \\
\hline 14 & $30 \cdot 1$ & FM & 15.9 & - & 15.9 & 3.94 & 3.91 & 3.89 & 3.92 & 0.05 & -0.01 \\
\hline 15 & $37 \cdot 8$ & MF & $21 \cdot 6$ & - & $21 \cdot 6$ & $4 \cdot 11$ & $4 \cdot 13$ & $4 \cdot 28$ & $4 \cdot 28$ & $-0 \cdot 17$ & -0.15 \\
\hline 16 & 38.9 & FM & $27 \cdot 0$ & 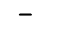 & $27 \cdot 0$ & $4 \cdot 69$ & $5 \cdot 18$ & $4 \cdot 10$ & 4.01 & 0.59 & $1 \cdot 17$ \\
\hline
\end{tabular}

^No data, due to aphakia.

$A=$ twin with longest duration of IDDM (years); $B=$ twin with shortest duration of IDDM (years), or no IDDM $(-) ; A-B=$ intrapair difference in diabetic duration (years); $R(A)=$ lens thickness $(\mathrm{mm})$, right eye, twin $A ; L(A)=$ lens thickness $(\mathrm{mm})$, left eye, twin $A ; R(B)=$ lens thickness $(\mathrm{mm})$, right eye, twin $B ; L(B)=$ lens thickness ( $\mathrm{mm})$, left eye, twin $B ; R$ dif=intrapair difference in lens thickness $(\mathrm{mm})$, right eye; Ldif=intrapair difference in lens thickness $(\mathrm{mm})$, left eye.

\section{MEASUREMENT OF LENS THICKNESS}

Lens thickness was measured by ultrasonography on a Teknar Ophthasonic Auto A Scan. All lenses were checked for a cataract by slitlamp examination in mydriasis, and photographs of the lenses in retroillumination were taken for registration of lens opacites. There was no actual cataract formation in any of the patients. Ten of the diabetic twins and two of the non-diabetic twins had a few vacuoles peripherally in the lens in both eyes. Speed of ultrasound was set to $1550 \mathrm{~m} / \mathrm{s}$ for aqueous and vitreous, and $1641 \mathrm{~m} / \mathrm{s}$ for the lens. The ultrasound device was calibrated on a special probe several times during the investigation period. All measurements were made on the same device and by the same trained ophthalmologist (NL). Only measurements with narrow and well defined echoes of equal height from anterior and posterior lens surfaces and with no internal spikes, as well as well defined echo from the vitreoretinal interface were accepted to obtain correct measurement of lens thickness and to ensure correct alignment and axial direction of the measurements. The mean of three measurements was taken, and all measurements were made 30 minutes after cycloplegia obtained by installation of one drop of cyclopentolate hydrochloride $1 \%$ and one drop of phenylephrine hydrochloride $10 \%$ in each eye twice, with an interval of 10 minutes.

\section{ANALYSIS OF DATA}

Within twin pairs, the partner with longer duration of IDDM was labelled ' $A$ ', and the partner with shorter duration or no IDDM was labelled ' $B$ '. Unaffected twins were assigned the value of 0.0 years of IDDM duration. Within each twin pair the difference in IDDM duration was calculated as the difference in duration between twin ' $A$ ' and twin ' $B$ ' $(A-B)$. The corresponding intrapair differences in lens thickness have been calculated for right and left eye separately. Standard procedures for regression analysis have been employed to obtain estimates of the correlation coefficients and regression coefficients (slopes) for right and left eye independently, and with $M Z$ twin pairs, DZ twin pairs of same sex (ss), and of opposite sex (os). The statistical test of differences between two correlation and regression coefficients was performed as suggested in Documenta Geigy. ${ }^{21}$

\section{Results}

Measurements of lens thickness were obtained from 92 right and 91 left eyes. One pair of $M Z$ twins did not want to have their pupils dilated, and one 12-year-old $\mathrm{MZ}$ twin and one 15-yearold DZss twin could not cooperate with lens thickness measurements. Further, one DZos twin had an aphakic left eye due to ocular trauma.

Complete ultrasonic measurements of lens thickness for both eyes of both cotwins were thus obtained in $15 \mathrm{MZ}$ pairs, $14 \mathrm{DZss}$ pairs, and $15 \mathrm{DZ}$ os pairs. In addition, measurements 


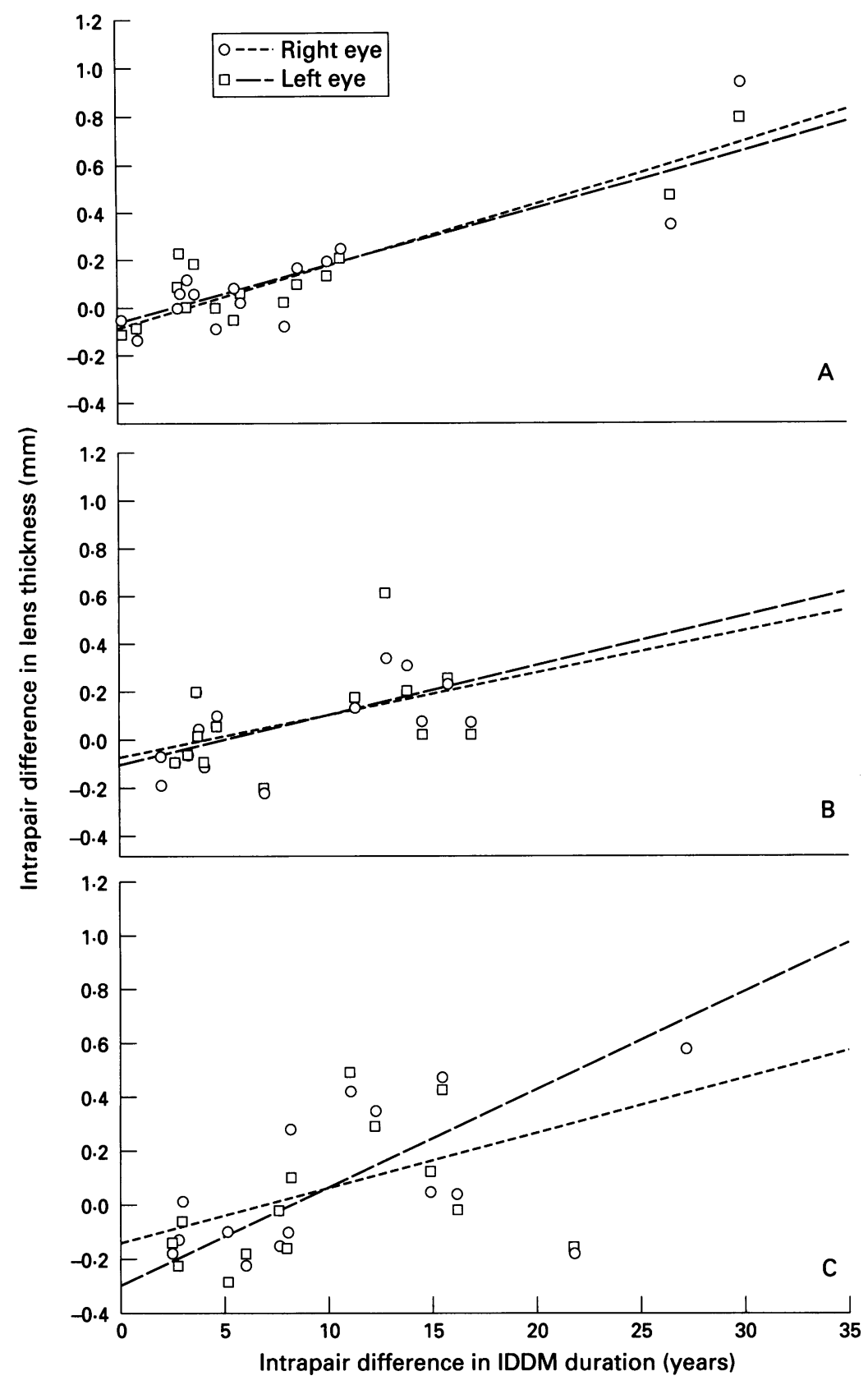

Figure 1 Scatterplot of intrapair difference in insulin dependent diabetes mellitus (IDDM) duration versus intrapair difference in lens thickness. (A) Monozygotic twin pairs; $(B)$ dizygotic twin pairs of same sex; $(C)$ dizygotic twin pairs of opposite sex.

of lens thickness from the right eye of one pair of DZos pair were included.

Age, sex, duration of diabetes, and lens thickness in the three twin groups can be seen in Table 2. In the $M Z$ group six pairs were concordant, in the DZss group no pair, and in the DZos group one pair was concordant with respect to diabetes.

Table 3 Regression analysis and correlation coefficients of intrapair difference in duration of IDDM versus intrapair difference in lens thickness

\begin{tabular}{llllllllr}
\hline Twins & Eye & Slope & $95 \% C I$ & Intercept & $95 \% C I$ & $\mathrm{r}$ & $95 \% C I$ & p Value \\
\hline $\mathrm{MZ}$ & $\mathrm{R}$ & 0.026 & $0.018 ; 0.035$ & -0.077 & $-0.177 ; 0.024$ & 0.879 & $0.667 ; 0.959$ & $<0.001$ \\
& $\mathrm{~L}$ & 0.024 & $0.017 ; 0.031$ & -0.050 & $-0.133 ; 0.033$ & 0.898 & $0.714 ; 0.966$ & $<0.001$ \\
DZss & $\mathrm{R}$ & 0.017 & $0.002 ; 0.033$ & -0.076 & $-0.227 ; 0.074$ & 0.581 & $0.073 ; 0.850$ & 0.029 \\
& $\mathrm{~L}$ & 0.020 & $-0.000 ; 0.041$ & -0.105 & $-0.307 ; 0.098$ & 0.527 & $0.000 ; 0.826$ & 0.053 \\
DZos & $\mathrm{R}$ & 0.024 & $0.005 ; 0.043$ & -0.193 & $-0.431 ; 0.044$ & 0.580 & $0.118 ; 0.836$ & 0.018 \\
& $\mathrm{~L}$ & 0.036 & $0.013 ; 0.059$ & -0.279 & $-0.566 ; 0.008$ & 0.688 & $0.257 ; 0.884$ & 0.005 \\
$\mathrm{DZ}$ & $\mathrm{R}$ & 0.021 & $0.001 ; 0.033$ & -0.137 & $-0.272 ;-0.033$ & 0.563 & $0.254 ; 0.768$ & 0.001 \\
& $\mathrm{~L}$ & 0.030 & $0.015 ; 0.044$ & -0.200 & $-0.363 ;-0.037$ & 0.634 & $0.348 ; 0.812$ & $<0.001$
\end{tabular}

$M Z=$ monozygotic twin pairs; DZss=dizygotic twin pairs of same sex; DZos=dizygotic twin pairs of opposite sex; $\mathrm{DZ}=$ dizygotic twin pairs combined; $\mathrm{R}=$ right eye; $\mathrm{L}=$ left eye; $\mathrm{CI}=$ confidence interval; $r=$ correlation coefficient.
The intrapair differences in duration of diabetes $(A-B)$ as well as the corresponding intrapair differences in lens thickness for right (Rdif) and left (Ldif) eye are also shown in Table 2. In the group of DZss twins, where there were no concordant pairs, the intrapair difference in diabetic duration is identical to the diabetic duration for the cotwin with diabetes. In order to estimate the influence of diabetes duration on lens thickness, the intrapair differences were plotted for each of the three groups shown in Figure 1. Regression lines for left and right eye are shown separately.

The slopes and intercepts for the regression analysis, with $95 \%$ confidence intervals, as well as correlation coefficients with $p$ values, are shown in Table 3.

There is a clear positive relation between increasing duration of diabetes and lens thickness, especially among the $M Z$ twins, where the slopes for right and left eyes are almost identical, and highly statistically significantly different from zero. In the DZss and DZos groups the slopes vary more, and the lower limits for $95 \%$ confidence intervals are close to zero, and one of them not statistically significantly positive (DZss left eye). After testing that the slopes, intercepts, and correlation coefficients for DZss and DZos, right and left eyes respectively, are not statistically significantly different, we have calculated the combined values (DZ) shown in Table 3. The intercepts are not statistically significantly different from zero in any of the groups, implying that it seems reasonable to look at influence of IDDM duration on lens thickness only from the clinical onset of IDDM. The correlation coefficients are highly statistically significant in the $M Z$ group, and significantly higher than the correlation coefficients in the $\mathrm{DZ}$ group $(p=0.034$ and $p=0.041$, for right and left eye respectively).

\section{Discussion}

Our study has confirmed previous studies reporting a positive association between diabetes and lens thickness. ${ }^{1617}$ The estimated correlations in our analyses seem to be higher than previously reported. This may be due to the application of the twin control design, by which the influence of confounding factors has been eliminated, or at least reduced. Our sample of twin pairs is small, but considered to be representative. It is particularly important that the distribution between $\mathrm{MZ}, \mathrm{DZss}$, and DZos pairs roughly equals $1: 1: 1$ which is expected according to principles of population genetics. ${ }^{19}$

The validity of assigning the value of 0 to the diabetes duration in unaffected twins relies upon the assumption that the effect of IDDM on lens thickness is exerted only from the clinical onset of IDDM. This seems to be a realistic assumption because of the belief that the increased lens thickness in IDDM patients is caused by the effect of hyperglycaemia which first manifests immediately preceding clinical onset in most IDDM patients. The fact that all estimated intercepts did not differ statistically 
significantly from 0,0 provides further support for the validity of the assumption.

The high degree of correlation found in our study may partly be explained by a few outlying values. Therefore, we have repeated the analyses after exclusion of such outliers. The point estimates of the slopes were only slightly changed and maintained the level of statistical significance; however, the difference in the correlation coefficients between $M Z$ and the combined DZ groups did not reach statistical significance, probably due to the small sample size. Therefore, the exclusion of outliers did not change our conclusion qualitatively. The almost similar slopes in the group of $M Z$ pairs compared with the group of $\mathrm{DZ}$ pairs suggest that the effect of IDDM duration on lens thickness is similar for $\mathrm{MZ}$ twins and $\mathrm{DZ}$ twins, thus independent of genetic factors. The higher correlation coefficients in $M Z$ twin pairs compared with $\mathrm{DZ}$ twin pairs suggest that, when adjusting for the effect of IDDM, there is an additional genetic component that determines lens thickness. Since there were no statistically significant differences in correlation coefficients between the $\mathrm{DZ}$ groups of same sex and opposite sex, there seems to be no influence of sex on the effect of diabetes on lens thickness. The design of the present study does not permit further analyses and conclusions on this aspect, since this requires investigation on a representative sample of non-diabetic $\mathrm{MZ}$ and $\mathrm{DZ}$ pairs.

Since the twin pairs included in our study are young and affected exclusively with IDDM we cannot generalise our findings from IDDM to non-insulin dependent diabetes mellitus (NIDDM). Previous studies have demonstrated that the type of diabetes was of importance for lens thickness. In IDDM a significant relation between duration of diabetes and lens thickness was found, ${ }^{17}$ whereas in NIDDM no relation between lens thickness and duration of diabetes was found. ${ }^{18}$ The duration effect in IDDM on lens thickness could be caused by accumulated damage by oxidation or non-enzymatic glycosylation involved in the regulatory mechanisms in the lens membrane. ${ }^{22} 23$ There is also evidence that different insulin-like growth factors could stimulate lens fibroblasts. ${ }^{24-26}$ Further, it has been speculated that retina derived growth factors may be responsible for lens hyperplasia and hypertrophy. ${ }^{27}$ This also agrees with the finding that lens thickness is associated with proliferative diabetic retinopathy. ${ }^{17}$ Simple osmotic swelling of the lens may be responsible for the short term alterations in refraction seen with high blood glucose levels where part of the glucose is transformed via the sorbitol pathway, but does not explain the strong relation that we found between duration of IDDM and lens thickness. In our study of young diabetic patients with generally short duration of diabetes only two developed proliferative retinopathy; consequently we could not investigate the possible association between IDDM duration, biometry of the lens, and diabetic retinopathy. We intend, however, in future follow up studies of the diabetic twin pairs in the Danish twin register to throw further light on these aspects.

This study has been supported financially by The Danish Diabetes Association; The Danish Eye Research Foundation (Øjenfonden); and Hotelejer Carl Larsen og Hustru Nicoline Larsens Fond (Værn om Synet).

We also want to thank the twins for their kind cooperation.

1 Sorsby A, Sheridan M, Leary GA. Refraction and its components in twins. Medical Research Council Special ponents in twins. Medical Research Council Spe
Report Series (303th edn). London: HMSO, 1962

2 Lin LI, Chen CJ. Twin study on myopia. Acta Genet Med Gemellol Roma 1987; 36: 535-40.

3 Teikari JM, Kapiro J, Koskenvuo MK, Vannas A. Heritability estimate for refractive errors - a populationbased sample of adult twins. Genetic Epidemiology 1988; 5: 171-81.

4 Teikari J, Koskenvuo M, Kaprio J, O'Donnell J. Study of gene-environment effects on development of hyperopia: a study of 191 adult twin pairs from the Finnish twin cohort study. Acta Genet Med Gemellol Roma 1990; 39: 133-6.

5 Teikari JM, Kaprio J, Koskenvuo M, O'Donnell J. Heritability of defects of far vision in young adults - a twin Heritability of defects of far vision in you
study. Scand $\mathcal{F}$ Soc Med 1992; 20: 73-8.

6 Goldschmidt E. On the etiology of myopia. Acta Ophthalmol (suppl 98) 1968.

7 Goldschmidt E. The importance of heredity and environment in the etiology of low myopia. Acta Ophthalmol 1981; 59: 759-62.

8 Fledelius HC, Fuchs J, Reck A. Refraction in diabetics during metabolic dysregulation, acute or chronic. With special reference to the diabetic myopia concept. Acta Ophthalmol Copenh 1990; 68: 275-80.

9 Gwinup G, Villarreal A. Relationship of serum glucose concentration to changes in refraction. Diabetes 1976; 25: 29-31.

10 Saito Y, Ohmi G, Kinoshita S, Nakamura Y, Ogawa K, Harino $S$, et al. Transient hyperopia with lens swelling at initial therapy in diabetes. Br $\mathcal{f}$ Ophthalmol 1993; 77: 145-8.

11 Planten JT, Kooijman AC, de Vries B, Woldringh J. Pathological-optic approach of cataract and lens. Ophthalmologica 1978; 176: 331-4.

12 Eva PR, Pascoe PT, Vaughan DG. Refractive change in hyperglycaemia: hyperopia, not myopia. $\mathrm{Br} f$ Ophthalmol 1982; 66: 500-5.

13 Fledelius HC. Refractive change in diabetes mellitus around onset or when poorly controlled. A clinical study. Acta Ophthalmol Copenh 1987; 65: 53-7.

14 Fledelius HC. Is myopia getting more frequent? A crosssectional study of 1416 Danes aged 16 years + . Acta Ophthalmol Copenh 1983; 61: 545-59.

15 Sjølie AK. Ocular complications in insulin-treated diabetes mellitus. Acta Ophthalmol (Copenh) (suppl 172) 1985

16 Fledelius HC, Miyamoto K. Diabetic myopia - is it lensinduced? An oculometric study comprising ultrasound measurements. Acta Ophthalmol Copenh 1987; 65: 469-73.

17 Sparrow JM, Bron AJ, Brown NA, Neil HA. Biometry of the crystalline lens in early-onset diabetes. Br $\mathcal{F}$ Ophthalmol 1990; 74: 654-60.

18 Sparrow JM, Bron AJ, Phelps Brown NA, Neil HA. Biometry of the crystalline lens in late onset diabetes: the importance of diabetic type. $\mathrm{Br} \mathcal{F}$ Ophthalmol 1992; 76: 428-33.

19 Kyvik KO, Green A, Beck-Nielsen H. The new Danish twin register: establishment and analysis of twinning rates. Int $\mathcal{f}$ Epidemiol 1995; 24: 589-96.

20 Kyvik KO, Green A, Beck-Nielsen H. Concordance rates of insulin-dependent diabetes mellitus: a population based insulin-dependent diabetes mellitus: a population

21 Documenta Geigy. Mathematics and Statistics. Section A. Basel: Ciba-Geigy, 1970

22 Garner MH, Spector A. ATP hydrolysis kinetics in $\mathrm{Na}$, K-ATPase in cataract. Exp Eye Res 1986; 42: 339-48.

23 Duncan G. Role of membranes in controlling ion and water movements in the lens. In: Elliot $\mathrm{K}$, Fitzsimons DW, eds. The human lens in relation to cataract. Basel: Ciba Foundation symposium 19, 1973: 99-116.

24 Reddan JR, Wilson-Dziedzic D. Insulin growth factor and epidermal growth factor trigger mitosis in lenses cultured in a serum-free medium. Invest Ophthalmol Vis Sci 1983; 24: 409-16.

25 Chamberlain CG, McAvoy JW. Evidence that fibroblast growth factor promotes lens fibre differentiation. Curr Eye growth factor promotes

26 Beebe DC, Silver MH, Belcher KS, van Wyk JJ, Svoboda $M E$. Lentropin, a protein that controls lens fibre formation, is related functionally and immunologically to the insulin like growth factors. Proc Natl Acad Sci USA 1986; 84: 2723-31.

27 Merimee TJ, Zapf J. Froesch ER. Insulin-like growth factors. Studies in diabetics with and without retinopathy. N Engl $\mathcal{Y}$ Med 1983; 309: 527-30. 